\title{
Can early closure and restenosis after endoluminal stenting be predicted from clinical, procedural, and angiographic variables at the time of intervention?
}

Eric Eeckhout, Guy van Melle, Jean-Christophe Stauffer, Pierre Vogt, Lukas Kappenberger, Jean-Jacques Goy

\begin{abstract}
Objectives-To develop a statistical model to assess the risk of early closure and restenosis on the basis of the information available at the time of stent implantation.

Design-An exploratory forward, stepwise multivariate logistic regression for each adverse event and multivariate polychotomous analysis for both events.

Setting-Tertiary referral centre for interventional treatment of coronary artery disease.

Patients-243 consecutive, successful stenting procedures between 1986 and 1993 with the Wallstent, the PalmazSchatz and Wiktor stents with analysis of clinical, procedural, and angiographic variables.

Mean outcome measures-Early closure was defined as angiographically documented stent thrombosis within the first 3 weeks after implantation and restenosis according to the $50 \%$ reference diameter reduction criterion.
\end{abstract}

Results-Overall early closure and restenosis rates were $14 \cdot 4 \%(35 / 243)$ and $19 \cdot 2 \%(40 / 208$, for a $97 \%$ repeat angiography rate). The statistical model predicted a worse outcome for male patients, with less restenosis in female patients. The only risk factor in female patients was the presence of collaterals to the target lesion. For male patients the following risk factors for closure and restenosis were retained: multiple stent implantation during the same session, the presence of collaterals to the target lesion, stenting of the left anterior descending artery or of the left circumflex artery, and bailout stenting. Only bailout stenting implied a decreased restenosis risk.

Conclusions-Clinical, procedural and angiographic variables increase the risk for early closure and restenosis after endoluminal stenting. The prediction models described above need to be validated prospectively.

(Br Heart f 1995;74:592-597)

Keywords: risk factors; stenting; restenosis; early closure
Endoluminal implantation of metallic stents was introduced in 1986 as an alternative to conventional percutaneous transluminal coronary angioplasty (PTCA) for the treatment of abrupt closure and restenosis after PTCA. ${ }^{1}$ Despite 8 years of clinical investigation and the promising results of the Benestent and STRESS trials, which favoured stenting over conventional balloon angioplasty for the treatment of de novo, focal stenoses in large native coronary arteries, reservations about stenting remain. ${ }^{23}$ These stem from the risk of early stent thrombosis: though its incidence has dropped dramatically from an initial rate of $20 \%$ to a recent rate of $3.5 \%$, thrombosis remains the chief drawback of stenting. ${ }^{34}$ In addition, even if endoluminal stenting does reduce the process of restenosis it does not prevent it.

There are few studies of the risks of closure or restenosis after intracoronary stenting: only one study and a few abstracts on closure and four on restenosis, with different results and various statistical methods. The purpose of the present study was to develop a statistical model to predict and quantify the risk factors for both early closure and restenosis after endoluminal stenting at the time of intervention. This requires a large study population with appropriate clinical and angiographic follow up, comparable management before and after the procedure, consistent inclusion criteria during the study years and a high angiographic follow up rate-conditions best complied with in a single centre. We therefore analysed various clinical, procedural, and angiographic variables observed in consecutively stented patients during a 7 year study period and investigated their possible contribution to early closure and restenosis.

\section{Patients and methods}

STUDY POPULATION

Consecutive successful stenting procedures in patients with angina pectoris treated between April 1986 and August 1993 were studied. Patients were considered (or on occasion reconsidered during follow up) for stenting in native coronary arteries and saphenous vein grafts when there was (a) abrupt or threatened vessel closure after PTCA with reduced distal vessel perfusion and impending infarction, (b) restenosis after initially successful PTCA, 
(c) de novo stenosis. Myocardial ischaemia had to be confirmed by either exercise testing or electrocardiographic modifications. Stenting was not performed when there was a contraindication to antiplatelet or anticoagulation treatment, $(b)$ in patients likely to be lost to follow up, (c) for lesions that were not suitable for stenting (ostial stenoses, large visible thrombus, extreme vessel tortuosity, vessels estimated $<3 \mathrm{~mm}$, preexisting chronic total occlusion for more than one month). The proposed indications were accepted by the hospital ethics committee and informed consent was obtained from each patient before implantation.

STENT TYPES, IMPLANTATION AND FOLLOW UP Three types of stents were used. The self expanding Wallstent (Medinvent, Lausanne) was implanted initially and until 1990 . Two balloon expandable stents were studied: the articulated Palmaz-Schatz stent (Johnson \& Johnson Interventional, Warren, NJ) and the Wiktor stent (Medtronic, Minneapolis, MN). All stents and their technique of implantation have been described previously. ${ }^{1-6}$

Before intervention all patients received aspirin ( $500 \mathrm{mg}$ four times a day), nifedipine (20 mg, sustained release), and a bolus of 15000 units of heparin. Procedures were performed with 9, 8, and 6 French guiding catheters. Prior balloon angioplasty (for bailout cases) or predilatation (for elective stenting) was carried out with conventional on-a-wire or monorail systems. In cases of acute or threatened closure after PTCA, prolonged conventional or autoperfusion balloon angioplasty could be performed at the discretion of the operator. Finally, if bailout stenting was needed, the operator was asked to strive towards an optimal angiographic result with complete sealing of the dissection. On occasion this required the implantation of several stents.

After intervention, introducers were pulled out at 4-24 $\mathrm{h}$ and after temporary discontinuation of a heparin drip titrated in order to obtain twice the normal value of the activated partial thromboplastin time. Oral anticoagulation (acenocoumarol) and antiplatelet treatment (aspirin ( $\geqslant 100 \mathrm{mg}$ four times a day), dipyridamole ( $75 \mathrm{mg}$ three times a day), sulphinpyrazone (600 $\mathrm{mg}$ between 1988 and 1990)), and a calcium channel blocker were started on the day of the procedure and continued up until a scheduled and mandatory repeat angiography at 6 months follow up. Treatment with heparin was stopped once a stable anticoagulation regimen was established, and before patients were discharged.

To prevent vascular complications at the puncture site, patients rested in bed for at least 3 days after the intervention. If vascular complications did not respond to conservative management (recompression) surgical repair was performed 4 hours after temporarily stopping the heparin infusion without any other modification or breach of the anticoagulation protocol.

During follow up, patients had repeat angiography at 6 months and as clinically required.

All patients received oral anticoagulation and full antiplatelet treatment during the first 6 months after intervention. Later only aspirin was given. Other cardiovascular drugs were prescribed for recurrence of angina or for other reasons such as hypertension.

\section{CRITICAL EVENTS}

Early closure was defined as a documented (by repeat angiography) stent thrombosis within the first 3 weeks after implantation.

Restenosis was defined as a $50 \%$ or more reduction of the reference diameter at 6 month repeat angiography (the binary restenosis criterion) measured by quantitative angiographic analysis.

\section{CLINICAL, PROCEDURAL, AND ANGIOGRAPHIC} VARIABLES

We studied clinical variables (age, sex, and angina functional class (New York Heart Association classification) before the intervention); procedural variables (stent size, stent length, delay between the last previous PTCA and stent implantation, number of previous PTCAs, indication for stenting (saphenous vein graft stenting, restenosis treatment, bailout stenting, elective stenting), stent type (Wallstent, articulated Palmaz-Schatz or Wiktor stent), multiple implant (the implantation of more than one stent in the same vessel during the same procedure), vessel type (left anterior descending artery (LAD), left circumflex artery (LCx), right coronary artery (RCA), and saphenous vein graft (SVG)), use of an autoperfusion balloon before stenting, temporary modification of the anticoagulation protocol (in case of bleeding)); and angiographic variables (stenosis length, vessel reference diameter, preprocedural minimal luminal diameter (MLD), preprocedural percentage stenosis, postprocedural MLD, postprocedural percentage stenosis, 6 months follow up MLD, 6 months follow up percentage stenosis, acute gain (postprocedural MLD minus preprocedural MLD), stent vessel mismatch (the difference between the chosen stent diameter and the measured reference diameter), run off (according to the TIMI classification and judged before conventional balloon angioplasty or elective stenting), lesion localisation (proximal, mild, or distal, according to the CASS Registry localisation protocol), the presence of collaterals to the target lesion (delayed retrograde filling of the target vessel beyond its stenosis during contrast injection)).

Quantitative angiographic analysis was performed by an independent operator, using the "worst" view. Most (74\%) angiographic films were analysed by means of the ANCOR system, while the remaining films were examined with the CASS system. ${ }^{7}$ This ANCOR system, derived from the CAAS algorithm, has been introduced and validated previously. ${ }^{8}$ Care was taken to produce films of good quality with full contrast filling of the stented segment and to routinely administer a vasolytic agent before the procedure. 
Table 1 Clinical characteristics

\begin{tabular}{ll}
\hline Characteristic & \\
\hline Mean age (yr) & $59(95 \%$ CI 57-60) (median 60) \\
Number of procedures: & 206 \\
Men & 37 \\
Women & \\
Angina class (NYHA): & 23 \\
2 & 100 \\
3 & 120 \\
4 & \\
\hline
\end{tabular}

NYHA, New York Heart Association classification.

Table 2 Procedural characteristics

\begin{tabular}{lccc}
\hline Characteristic & Mean & $95 \% C I$ & Median \\
\hline Stent size & $3 \cdot 7 \mathrm{~mm}$ & $3 \cdot 6$ to $3 \cdot 8$ & $3 \cdot 5$ \\
Stent length & $19 \mathrm{~mm}$ & 18 to 19 & 15 \\
Delay between last previous PTCA & 7 months & $4 \cdot 7$ to $9 \cdot 2$ & 5 \\
and stent implantation & Number of procedures & \\
& & \\
Previous PTCA (n): & 44 & \\
1 & 27 & \\
2 & 8 & \\
3 & 6 & \\
4 & 85 & \\
Total & 59 & \\
Indication: & 51 & \\
Saphenous vein graft & 31 & \\
Bailout stenting & 40 & \\
LAD & 16 & \\
RCA & 3 & \\
LCx & 90 & \\
LC & & \\
Total & 36 & \\
Restenosis treatment & 24 & \\
LAD & 7 & \\
RCA & 67 & \\
LCx & & \\
Total & 25 & \\
De novo stenosis & 2 & \\
RCA & 27 & \\
LCx & 133 & \\
Total & 69 & \\
Stent types: & $183 / 60$ & \\
Wallstent & 67 & \\
Palmaz-Schatz & 25 & \\
Wiktor & 3 & \\
Single/multiple implant & & \\
Vessel type: & & \\
LAD & & \\
RCA & & \\
LCx & & \\
LM & & \\
\hline
\end{tabular}

95\%CI, 95\% confidence interval; PTCA, percutaneous transluminal coronary angioplasty; $\mathrm{LAD}$, left anterior descending artery; RCA, right coronary artery; LCx, left circumflex branch; LM, left main.

Table 3 Angiographic characteristics

\begin{tabular}{|c|c|c|c|}
\hline Characteristic & Mean & $95 \% C I$ & Median \\
\hline $\begin{array}{l}\text { Stenosis length } \\
\text { Reference diameter } \\
\text { Pre MLD } \\
\text { Pre \% stenosis } \\
\text { Post MLD } \\
\text { Post \% stenosis } \\
\text { Follow up MLD } \\
\text { Follow up \% stenosis } \\
\text { Acute gain } \\
\text { Mismatch }\end{array}$ & $\begin{array}{c}9.54 \mathrm{~mm} \\
3.37 \mathrm{~mm} \\
1.10 \mathrm{~mm} \\
67 \% \\
2.72 \mathrm{~mm} \\
23 \% \\
2.06 \mathrm{~mm} \\
42 \% \\
1.62 \mathrm{~mm} \\
0.64 \mathrm{~mm}\end{array}$ & $\begin{array}{l}9.02 \text { to } 10.06 \\
3.28 \text { to } 3.47 \\
1.03 \text { to } 1.17 \\
65 \text { to } 69 \\
2.58 \text { to } 2.86 \\
22 \text { to } 25 \\
1.92 \text { to } 2.20 \\
38 \text { to } 46 \\
1.48 \text { to } 1.77 \\
0.56 \text { to } 0.71\end{array}$ & $\begin{array}{c}9 \cdot 20 \\
3 \cdot 30 \\
1 \cdot 00 \\
67 \\
2 \cdot 60 \\
22 \\
2 \cdot 30 \\
32 \\
1 \cdot 50 \\
0 \cdot 50\end{array}$ \\
\hline \multicolumn{4}{|c|}{ Number of procedures } \\
\hline $\begin{array}{l}\text { Run off (TIMI): } \\
0 \\
1 \\
2 \\
3\end{array}$ & $\begin{array}{r}9 \\
6 \\
2 \\
226\end{array}$ & & \\
\hline $\begin{array}{l}\text { Site: } \\
\text { Proximal } \\
\text { Mid } \\
\text { Distal } \\
\text { Collaterals (yes/no) }\end{array}$ & $\begin{array}{l}93 \\
138 \\
12 \\
15 / 228\end{array}$ & & \\
\hline
\end{tabular}

95\%CI, 95\% confidence interval; Pre, preprocedural; Post, postporcedural; MLD, minima luminal diameter; mismatch, stent-vessel reference diameter mismatch.

\section{STATISTICAL ANALYSIS}

Values are presented as means with their $95 \%$ confidence intervals and medians. Discrete variables were compared by means of a $\chi^{2}$ test.

The study population was categorised with respect to the occurrence of early closure and/or restenosis and secondarily according to the clinical, procedural, and angiographic variables. Before analysis, continuous variables were split at the median and discrete variables were dichotomised when there were more than 2 subgroups. Effects were regarded as significant when the null hypothesis could be rejected at a $5 \%$ probability. Continuous variables are presented as means with their $95 \%$ confidence intervals and medians.

Initially, early closure and restenosis were studied separately and we screened for risk factors first by forward, stepwise multivariate regression analysis. All procedures, were considered when early closure was analysed. However, restenosis could not be or was not studied in patients who experienced early closure (permanent or temporary (because of successful recanalisation)) or who refused repeat angiography.

A second step consisted of a multivariate polychotomous logistic regression analysis that considered both closure and restenosis. This model permits the prediction at the time of intervention of both critical events. The rationale for this analysis was the assumption that patients who experience early closure may behave differently from "no closure" patients in terms of the late phenomenon of restenosis. Therefore each patient was regarded as being subject to closure and restenosis.

The statistical package (SAS) was used on a VAX-computer.

\section{Results}

PATIENT POPULATION

Between April 1986 and April 1993, 243 stenting procedures (in a total of 215 patients) were performed at our institution. Tables 1-3 show the clinical, procedural, and angiographic variables. The different indications and target vessels were equally distributed among the different stent types $(P=0.08)$. Six patients refused repeat angiography and 27 had permanent stent thrombosis, so 210 cases were available for quantitative analysis of follow up angiograms (210/216 or $97 \%$ angiographic follow up rate).

CRITICAL EVENTS

Early closure occurred in 35 (14.4\%) out of 243 procedures. This phenomenon was subacute (after the first $24 \mathrm{~h}$ and before 3 weeks after implantation) in 28 cases.

Because of the stringent anticoagulation regimen bleeding complications at the puncture site were not uncommon. Thirty two patients $(13.2 \%)$ required surgical repair and interruption of the heparin perfusion. However, only two of these experienced subacute closure. Eight patients treated by autoperfusion balloon angioplasty for threatened closure during PTCA required bailout stenting. Yet, closure occurred in only one of these cases. 
Table 4 Risk factors for closure and restenosis

\begin{tabular}{lll}
\hline Variable & $\begin{array}{l}\text { Closure } \\
(\beta \text { coefficient }(S E))\end{array}$ & $\begin{array}{l}\text { Restenosis } \\
(\beta \text { coefficient }(S E))\end{array}$ \\
\hline Females: & & \\
$\quad$ Intercept & $-2.20(0.74)$ & $-3.09(0.98)$ \\
$\quad$ Collaterals & $1.42(0.97)$ & $1.01(1.46)$ \\
Males: & & $-1.29(0.32)$ \\
$\quad$ Intercept & $-3.68(0.64)$ & $1.39(0.53)$ \\
$\quad$ Bailout stenting & $0.81(0.50)$ & $1.00(0.43)$ \\
Multiple implant & $0.93(0.60)$ & $1.48(1.09)$ \\
Collaterals & $2.71(0.91)$ & $0.14(0.46)$ \\
LAD or LXx & & \\
$\quad$ stenting & $2.27(0.60)$ &
\end{tabular}

$\mathrm{LAD}$, left anterior descending artery; LCx, left circumflex artery

Angiographic restenosis was detected in 42 $(20 \%)$ of 210 procedures. Pure angiographic restenosis without angina recurrence was observed in seven cases at 6 month repeat angiography. All other cases presented with clinical restenosis.

There was no significant difference in the incidence of closure $(P=0 \cdot 27)$ and restenosis $(P=0.56)$ over the years.

We analysed risk factors for early closure for all procedures $(n=243)$, whereas only the 202 cases without closure at repeat angiography were analysed for restenosis risk factors: 40 had restenosis $(19 \cdot 8 \%)$. The six patients who refused repeat angiography had no clinical restenosis. Eight patients with early closure were successfully recanalised and two of these had restenosis. This closure subgroup was too small to be compared with the no closure group.

\section{FORWARD, STEPWISE MULTIVARIATE REGRESSION} Closure

The following risk factors were retained in a forward, stepwise regression model: (a) the presence of collaterals ( $60 \%$ with $v 11.4 \%$ without, $\mathrm{P}=0.0001)$, (b) stenting of the LAD (30\% with $v 3.4 \%$ without for SVG and $5.6 \%$ for RCA, P $=0.0002)$ or the LCx $(28 \%, P=$ $0.0001),(c)$ bailout stenting $(24.4 \%$ with $v$ $16.4 \%$ without, for restenosis treatment and $0 \%$ for de novo stenting of stenoses, $\mathrm{P}=0.03$ ), (d) age $>60$ years $(19.8 \%$ v $9.9 \%, \mathrm{P}=$ $0.0002),(e)$ stents $\leqslant 15 \mathrm{~mm}(16 \%$ v $12.6 \%, \mathrm{P}$ $=0.04)$, and $(f)$ Wallstent $(16.5 \% v 8.7 \%$ for the Wiktor and $17 \cdot 1 \%$ for the Palmaz-Schatz stents). The resulting model showed an $88 \cdot 7 \%$ concordance.

\section{Restenosis}

The following risk factors were significant: $(a)$ male sex $(22.4 \% v 6.3 \%, \mathrm{P}=0.04)$, (b) multiple implant $(32 \% v 15.9 \%, P=0.04),(c)$ Wiktor and Palmaz-Schatz stent $(21.7 \% v$ $18.2 \%$ for Wallstent, $\mathrm{P}=0.04$ ), (d) stenting other than bailout stenting $(25 \cdot 4 \%$ v $8 \cdot 8 \%$ for no bailout, $P=0.005)$. The resulting model showed a $66 \cdot 3 \%$ concordance.

\section{MULTIVARIATE POLYCHOTOMOUS LOGISTIC} REGRESSION

In view of a different outcome in female and male patients in this study we decided to apply the polychotomous model separately to men and women.

Table 4 shows different variables retained as risk factors for closure and restenosis. Table 5 shows the prediction of both critical events in men according to the presence of these risk factors.

\section{Discussion}

The primary success rate of percutaneous transluminal coronary angioplasty has improved dramatically since its introduction in $1977^{9}$ mainly because of the refinement lesion selection and the improvement in operator's skill, which has resulted in fewer technical failures. However, the incidence of the technique's two major shortcomings, acute abrupt closure and late restenosis, has remained unchanged. ${ }^{10}$ Endoluminal implantation of metallic stents has been performed in human coronary arteries since 1986 in an attempt to overcome both complications. From 1986 on, different stent types have been under clinical investigation. This first critical evaluation of these new devices in January 1991 was disappointing for the Wallstent, with an unacceptable high early stent thrombosis rate (up to $20 \%$ ), and promising for the Palmaz-Schatz stent with less closure $(3 \cdot 7 \%))^{45}$

This study examines risks factors for early closure and restenosis based on clinical, procedural, and angiographic variables observed in 243 successful stenting procedures. A thor-

Table 5 Prediction of early closure and restenosis in men

\begin{tabular}{|c|c|c|c|c|c|c|c|}
\hline \multirow[b]{2}{*}{ Risk factors } & \multicolumn{3}{|c|}{ Predicted risk (\%) } & \multicolumn{4}{|c|}{ Observed risk (\%) } \\
\hline & Event free & Closure & Restenosis & No of cases & Event free & Closure & Restenosis \\
\hline No factors present & $76 \cdot 9$ & 1.9 & $21 \cdot 1$ & 52 & $73 \cdot 1$ & 1.9 & $25 \cdot 0$ \\
\hline $\mathrm{L}$ & $64 \cdot 1$ & $15 \cdot 7$ & $20 \cdot 3$ & 32 & $62 \cdot 5$ & $18 \cdot 8$ & $18 \cdot 8$ \\
\hline C & $38 \cdot 6$ & $14 \cdot 7$ & $46 \cdot 6$ & 0 & - & - & - \\
\hline$M$ & $55 \cdot 4$ & $3 \cdot 5$ & $41 \cdot 1$ & 32 & $62 \cdot 5$ & 0 & $37 \cdot 5$ \\
\hline B & 88.9 & $5 \cdot 0$ & $6 \cdot 1$ & 24 & $91 \cdot 7$ & $8 \cdot 3$ & 0 \\
\hline $\mathrm{L}, \mathrm{C}$ & $16 \cdot 5$ & $60 \cdot 6$ & $22 \cdot 9$ & 5 & $40 \cdot 0$ & $60 \cdot 0$ & 0 \\
\hline $\mathrm{L}, \mathrm{M}$ & $40 \cdot 4$ & $24 \cdot 9$ & $34 \cdot 6$ & 2 & 0 & $100 \cdot 0$ & 0 \\
\hline L, B & $61 \cdot 5$ & $33 \cdot 6$ & $4 \cdot 9$ & 33 & $66 \cdot 7$ & $24 \cdot 2$ & $9 \cdot 1$ \\
\hline $\mathrm{M}, \mathrm{C}$ & $19 \cdot 1$ & $18 \cdot 4$ & $62 \cdot 5$ & 0 & - & - & - \\
\hline M, B & $75 \cdot 3$ & $10 \cdot 7$ & $14 \cdot 0$ & 8 & $75 \cdot 0$ & 0 & $25 \cdot 0$ \\
\hline C, B & $46 \cdot 4$ & $39 \cdot 5$ & $14 \cdot 0$ & 2 & 0 & $100 \cdot 0$ & 0 \\
\hline $\mathrm{B}, \mathrm{M}, \mathrm{L}$ & $38 \cdot 6$ & $53 \cdot 2$ & $8 \cdot 3$ & 6 & $16 \cdot 7$ & $83 \cdot 3$ & 0 \\
\hline B, M, C & $25 \cdot 2$ & $54 \cdot 2$ & $20 \cdot 6$ & 0 & - & - & - \\
\hline $\mathrm{M}, \mathrm{C}, \mathrm{L}$ & $7 \cdot 1$ & $66 \cdot 1$ & $26 \cdot 8$ & 2 & 0 & 0 & $100 \cdot 0$ \\
\hline B, C, L & $10 \cdot 5$ & $85 \cdot 9$ & $3 \cdot 6$ & 1 & 0 & $100 \cdot 0$ & 0 \\
\hline All factors present & $4 \cdot 4$ & $91 \cdot 4$ & $4 \cdot 2$ & 0 & - & - & - \\
\hline
\end{tabular}

L, LAD (left anterior descending artery) or LCx (left circumflex artery) stenting; $M$, multiple stent implantation; C, the presence of collaterals to the target lesion; $\mathrm{B}$, bailout stenting. 
ough search of published reports found one paper and a few abstracts on risk factors for early closure; none mentioned the statistical methods. The risk factors identified were thrombocytosis, stents smaller than $3 \mathrm{~mm}$, multiple Palmaz-Schatz stent implantation, and inability to stabilise the dissection by stenting in bailout cases. ${ }^{11-13}$ Only one paper, by Nath et al $(\mathrm{n}=145)$, identified lesion eccentricity and bailout stenting as risk factors for closure with the Gianturco-Roubin stent in a multivariate model. ${ }^{14}$

Risk factors for restenosis have been studied by Strauss et al (Wallstent, $\mathrm{n}=214,1991$ ), Carrozza et al (Palmaz-Schatz, $\mathrm{n}=250,1992)$, Ellis et al (Palmaz-Schatz, $\mathrm{n}=206,1992)$, and de Jaegere et al (Wiktor stent, $\mathrm{n}=91$ ). ${ }^{14-18}$ Strauss used the binary restenosis $(a \geqslant 50 \%$ reduction compared with the reference diameter) criterion (criterion 1) and a criterion suggested by their group (a loss of $\geqslant 0.72 \mathrm{~mm}$ in minimal luminal diameter at repeat angiography, criterion 2) and an odds ratio analysis. Risk factors according to criterion 1 and 2 were: multiple stenting and stent-vessel mismatch of $0.7 \mathrm{~mm}$ or more. They identified two other risk factors: vein graft stenting and postprocedural residual stenosis of $\geqslant 20 \%$. Their $95 \%$ confidence interval for the odds ratio, however, ranged from 0.91 to 2.67 , which makes their conclusions debatable. Carrozza et al retained three variables from univariate analysis (diabetes mellitus, LAD stenting, and a postprocedural MLD of $<3.3 \mathrm{~mm}$ ). They retained LAD stenting in a multivariate stepwise model. Ellis et al were the only investigators who removed early closures for their restenosis study. The risk factors tested in their multivariate logistic regression model were a history of restenosis, a postprocedural residual stenosis of $>0 \%$, and multiple stent implantation. Only multiple stent implantation was an independent risk factor in this study. Otherwise, a residual stenosis of less than $0 \%$ has not been found in our experience and is seldom reported in published studies. Finally, de Jaegere et al in 91 Wiktor stent lesions found a relative gain (the acute gain normalised to the vessel size) of $>0.48$ to be the only risk factor for restenosis according to the $0.72 \mathrm{~mm}$ late loss criterion.

In the present study, we decided to apply the binary restenosis criterion based on the generalised model for restenosis, proposed by Kuntz et al. ${ }^{19}$ One of the major criticisms of these investigators of the criterion of $0.72 \mathrm{~mm}$ late loss emerges from the present review of published reports. It has recently become apparent that a larger acute gain may be associated with more late loss of diameter. ${ }^{18}$ Patients with an excellent angiographic result were more likely to fit into the $0.72 \mathrm{~mm}$ late loss criterion of restenosis in the study by Strauss et al and in the more recent work of de Jaegere $e t$ $a l .{ }^{15} 18$

The purpose of the present study was to investigate the ability to predict both early closure and restenosis at the time of implantation. An exploratory multivariate logistic regression was first performed for each critical event in order to detect possible risk factors.

This exploratory analysis for early closure suggested that the Wallstent was more thrombogenic than the Palmaz-Schatz stent. Subanalysis of our data shows that this is caused by a higher thrombosis rate in cases treated by placing short ( $\leqslant 15 \mathrm{~mm}$ ) Wallstents (8 in 22 for short $v 14$ in 111 for long Wallstents). A possible explanation for this observation may be incomplete sealing of the dissection by the shorter Wallstent which may increase the risk of closure. Despite an apparent similar thrombosis rate for the Wallstent $(16.5 \%)$ and the Palmaz-Schatz stent $(17 \cdot 1 \%)$, the initial multivariate model predicts more closure with the Wallstent. This may be explained by the observed increased closure risk for stents $\leqslant 15 \mathrm{~mm}$ (which includes the Palmaz-Schatz stents, that can not be counted twice in the multivariate model). In the global polychotomous model the initial disadvantage for the Wallstent was lost because this stent tended to induce fewer restenosis.

Five variables were retained in the final polychotomous model as risk factors for early closure and restenosis: one clinical characteristic (male gender), three procedural variables (LAD or LCx stenting, bailout stenting, multiple stent implantation), and one angiographic variable (the presence of collaterals).

Women had a more favourable outcome with less restenosis (about three times less than men) and the only independent variable that can be kept in the polychotomous model is the presence of collaterals to the target lesion, which increases the risk of early closure.

This study shows that the LAD and LCx behave comparably and are identified as a risk factor, mainly for closure. The relative risk for closure after stenting of the LAD or $\mathrm{LCx}$ is eight times that for the RCA and SVG.

Multiple stent implantation chiefly increases the risk of restenosis. This finding accords with the results of Strauss $e t$ al and Ellis et al. Three independent investigators found that multiple stent implantation doubled the risk of restenosis. Also, bailout stenting was found to be a risk factor for closure; however, it reduced the risk of restenosis. The presence of collaterals, which has not been suggested as a risk factor before, emerged as a powerful predictor for both restenosis and closure. In general, it seems that early closure is easier to predict (higher $\beta$ coefficient) than restenosis. The suggested estimates for early closure and restenosis in table 5 can be validated in ongoing trials of endoluminal stenting. The model predicts $1.9 \%$ early closure and $21 \cdot 1 \%$ restenosis in the absence of risk factors. Two large multicentre trials comparing stenting with conventional angioplasty for de novo stenoses have recently been published: the STRESS trial (n $=205$ ) had a $3 \cdot 4 \%$ incidence of early closure; and repeat angiography at 6 months in the Benestent trial $(n=259)$ showed a restenosis rate (binary model) of $22 \% .^{23}$

\section{STUDY LIMITATIONS}

This report summarises experience of an evolving technique. 
- The suggested risk factors may not apply to heparin-coated stents, different deployment techniques (such as high pressure, non-compliant adjunctive angioplasty) and new postprocedural managements. Some groups that use these approaches no longer prescribe oral anticoagulation and have reported very low closure rates. ${ }^{20} 21$

- The subgroup of eight patients with temporary closure was too small to be studied.

- A limited group of variables was studied. Others may have been overlooked.

CONCLUSIONS AND CLINICAL IMPLICATIONS Several factors seem to influence early closure and restenosis at the time of stent implantation:

- Female patients have a more favourable outcome, with fewer restenosis, than men. The presence of collaterals to the target lesion is the only independent risk factor for closure in women.

- Restenosis and closure in men is affected by procedural factors: this paper confirms previous work that multiple stent implantation should be avoided as it doubles the risk for restenosis, stenting of the left anterior descending artery mainly increases the risk for early closure. Bailout stenting increases the risk for early closure but diminishes the risk for restenosis. Angiographic factors: the presence of collaterals to the target lesion is the strongest predictor for early closure and for restenosis.

This model has been developed for the Wallstent, the articulated Palmaz-Schatz, and Wiktor stents. Because it has been developed to predict events at the time of implantation, it may be of practical interest and suggest treatments other than endoluminal stenting in patients where several risk factors are present.

We hope that new, less thrombogenic stents will be developed and make this model superfluous.

1 Sigwart U, Peul J, Mirkowitch V, Joffre F, Kappenberger L. Intravascular stents to after trans

2 Fischman DL, Leon MB, Baim DS, et al. A randomized comparison of coronary-stent placement and balloon angioplasty in the treatment of coronary artery disease. NEngl F Med 1994;331:496-501.

3 Serruys PW, de Jaegere $\mathrm{P}$, Kiemeneij F, et al. A comparison of balloon-expandable-stent implantation with balloon angioplasty in patients with coronary artery disease. $N$ Engl f Med 1994;331:489-95.

4 Serruys PW, Strauss BH, Beatt KJ, et al. Angiographic follow up after placement of a self-expanding coronaryfollow up after placement of a self-expandin

5 Schatz RA, Baim DS, Leon MB, et al. Clinical experience with the Palmaz-Schatz coronary stents. Initial results of a with the Palmaz-Schatz coronary stents. Initial
multicentre study. Circulation 1991;83:148-61.

6 Buchwald A, Unterberg C, Werner G, Voth E, Kreuger H, Wiegand V. Initial results with the Wiktor stent: a new balloon-expandable coronary stent. Clin Cardiol 1991;14: 374-9.

7 Reiber JHC, Serruys PW, Kooijman CJ, et al. Assessment of short, medium, and long-term variations in arterial dimensions from computer-assisted quantitation of coronary cineangiograms. Circulation 1985;71:280-8.

8 Pope DL, Parker DL, Gustafson DE, Clayton PD. Dynamic search algorithms in left ventricular border recognition and analysis of coronary arteries. Comp Cardiol 1984;4:71-5.

9 Greunttig AR. Transluminal dilatation of coronary artery stenosis. Lancet 1978;i:263.

10 Detre K, Hulubkov R, Kelsey S, et al. Percutaneous transluminal coronary angioplasty in 1985-1986 and 1977-1981. N Engl f Med

11 Schatz RA, Leon MB, Baim DS, Ellis SG, Erbel R, Hirsschfeld JW. Balloon-expandable intracoronary stents: initial results of a multicentre experience [abstr]. Circulation 1989;80:II-174

12 Leon MB, Almagor Y, Erbel R, Teirstein PS, Perez J, Schatz RA. Subacute thrombotic events after coronary stent placement: clinical spectrum and predictive factors [abstr]. Circulation 1989;80:II-174.

13 Liu MW, Agrawal SK, Voorhees WD, et al. Risk stratification of stent thrombosis following intracoronary stentmulticentre registry study [abstr]. Circulation 1993;88: I-122.

14 Nath FC, Muller DWM, Ellis SG, et al. Thrombosis of a flexible coil coronary stent: frequency, predictors and clinical outcome. $\mathcal{f} \mathrm{Am}$ Coll Cardiol 1993;20: $622-7$.

15 Strauss BH, Serruys PW, de Scheerder IK, et al. Relative risk analysis of angiographic predictors of restenosis within the coronary Wallstent. Circulation 1991;84: 1636-43.

16 Carrozza JP, Kuntz RE, Levin MJ, et al. Angiographic and clinical outcome of coronary stenting: immediate and ong-term results from a large single-centre experience. $f$ Am Coll Cardiol 1993;20:328-37.

17 Ellis S, Savage M, Fischman D, et al. Restenosis after placement of Palmaz-Schatz stents in native coronary arteries. Circulation 1992;86:1836-44.

18 de Jaegere PPT, Serruys PW, Bertrand M, et al. Angiographic predictors of restenosis following Wiktor stent graphic predictors of restenosis following Wiktor stent implantation in

19 Kuntz RE, Gibson MC, Nobuyoshi M, Baim DS. Generalized model of restenosis after conventional balloon angioplasty, stenting and directional atherectomy. $f$ Am Coll Cardiol 1993;21:15-25.

20 Barragan P, Sainsous J, Silvestri M, et al. Ticlopidine and subcutaneous heparin as an alternative regimen following coronary stenting. Cathet Cardiovasc Diagn 1994;32: 133-8.

21 Colombo A, Hall P, Nakamura S, et al. Intracoronary stenting without anticoagulation accomplished with intravascular ultrasound guidance. Circulation 1995;91: 1676-88. 